\title{
ACTITUDES, PRÁCTICAS Y ESTILOS DE VIDA EN ADOLESCENTES DE INSTITUCIONES DE EDUCACIÓN SECUNDARIA DE LA CIUDAD DE POPAYÁN, 2016
}

Omar Andrés Ramos Valencia', Laura Ximena Bahos Ruano²,

Yuliana Buitron González², María Alejandra Jaimes²,

Paola Andrea Andrade ${ }^{2}$

\section{Resumen}

Objetivo: Determinar los estilos de vida de los entornos escolares, sus conocimientos, actitudes y prácticas hacia su salud, en los estudiantes de educación media vocacional en seis instituciones educativas públicas y privadas de la ciudad de Popayán. Materiales y métodos: Estudio descriptivo, observacional de corte transversal, con muestra probabilística seleccionando 135 estudiantes. Se aplicó un instrumento tipo encuesta para el análisis de factores sociodemográficos, actitudes, prácticas y hábitos de vida de los adolescentes. Resultados: De los estudiantes encuestados son de grado noveno (51,9\%); han fumado tabaco alguna vez (38,5\%); han consumido alcohol (88,9\%); han consumido sustancias psicoactivas (29,6\%); realizan actividad física (87,4\%); han iniciado su vida sexual (59,3\%). Conclusiones: los hábitos no saludables en los adolescentes se ven influenciados por la vulnerabilidad y entorno social, el uso de la tecnología y la vida moderna, los cuales afectan el tiempo libre y los determinantes sociales en salud.

Palabras clave: estilo de vida, factores de riesgo, hábitos, adolescentes, educación secundaria.

\footnotetext{
${ }^{1}$ Magíster en Salud Pública, Especialista en Auditoría en Salud, fisioterapeuta. Docente de la Universidad del Cauca, Programa Fisioterapia. Correo electrónico: omaramos@unicauca.edu.co

${ }^{2}$ Estudiantes del programa de Fisioterapia, Universidad del Cauca.
} 


\section{BEHAVIORS, PRACTICES AND LIFESTYLES IN HIGH SCHOOL TEENAGERS FROM POPAYAN, 2016}

Omar Andrés Ramos Valencia, Laura Ximena Bahos Ruano, Yuliana Buitron González, María Alejandra Jaimes, Paola Andrea Andrade

\section{Abstract}

Objective: To determine the lifestyles of school environments, their knowledge, attitudes and practices towards their health, in vocational secondary education students in six public and private educational institutions from Popayán. Materials and methods: Descriptive, observational cross -sectional study with a probabilistic sample, selecting 135 students, a survey- type instrument was used to analyze the sociodemographic factors, attitudes, practices and life customs of teenagers. Results: the respondent students, they were nineth grade $(51.9 \%)$; have had smoked cigarettes (38.5\%); consumed alcohol (88.9\%) and psychoactive substances (29.6\%); performed physical activity (87.4\%); having started their sexual life (59.3\%). Conclusions: Unhealthy habits in adolescents are influenced by vulnerability and social environment, the use of technology and modern life, which affect free time and social determinants in health. 


\section{ATITUDES, PRÁTICAS E ESTILOS DE VIDA EM ADOLESCENTES DAS INSTITUIÇÕES DE ENSINO MEIO NA CIDADE DE POPAYÁN, 2016}

Omar Andrés Ramos Valencia, Laura Ximena Bahos Ruano, Yuliana Buitron González, María Alejandra Jaimes, Paola Andrea Andrade

\section{Resumo}

Objetivo: determinar os estilos de vida no ambiente escolar, seus conhecimentos, atitudes e práticas para sua saúde, nos estudantes do ensino meio em seis escolas públicas e privadas na cidade de Popayán. Métodos: estudo descritivo, observacional de tipo transversal, com amostra probabilística selecionando 135 estudantes. Aplicou-se o instrumento tipo enquete para as análises dos fatores sociodemograficos, atitudes, práticas e estilos de vida dos adolescentes. Resultados: os alunos enquistados são do nono ano $(51,9 \%)$, tem fumado alguma vez $(38,5 \%)$, tem consumido álcool $(88,9 \%)$, tem consumido substâncias psicoativas $(29,6 \%)$, praticam atividade física $(87,4 \%)$, tem vida sexual ativa (59,3\%). Conclusões: os estilos de vida não saludáveis nos adolescentes tem influencia pela vulnerabilidade e entorno social, o uso da tecnologia, a vida moderna os quais afetam seu tempo livre e os determinantes sociais na saúde. 


\section{Introducción}

Las actitudes y prácticas de hábitos saludables de los adolescentes se pueden ver permeadas por la vulnerabilidad que estos presentan, entendida como la falta de protección de los grupos sociales causada por las inequidades en sus determinantes sociales (1). Esta vulnerabilidad puede tener diversos factores asociados como estrés, apego, resiliencia e inteligencia emocional, que según como sean tomados por el individuo pueden convertirse en factores de protección o riesgo. Cuando los adolescentes son vulnerables de forma emocional, económica o familiar, se aumenta su probabilidad de riesgo para el consumo de sustancias adictivas $(2,3)$.

Otro factor que aumenta el riesgo de consumo de sustancias psicoactivas (SPA) es la disponibilidad de estas, el fácil acceso y adquisición, muchas veces proporcionada por sus amigos en sus círculos sociales, o aceptadas socialmente como el caso del tabaco y el alcohol. Esta disponibilidad de sustancias adictivas para los adolescentes se vive desde los barrios más adinerados hasta los más pobres: los adolescentes buscan probar o curiosear nuevas formas de sentir, o simplemente mantener un estatus más alto o ser aceptado en algún grupo (4). Los adolescentes se encuentran expuestos a muchas tensiones dentro o fuera de sus hogares, siendo el rol de la familia el primer factor disparador de una problemática psicoemocional, y el círculo social el escapismo para enfrentar los problemas, encontrando en la droga o el alcohol un refugio. De tal manera que estudios demuestran que la influencia de los amigos, el deseo de imitar a los adultos y el proceso de socialización en su despertar sexual son los factores detonadores del inicio de consumo de psicoactivos $(5,6)$.

Por otro lado, con la aparición de nuevas tecnologías los adolescentes adquieren nuevas habilidades para manipular distintas herramientas tecnológicas y explorar un mundo que es virtual, intangible y digital, con ello se han efectuado modificaciones en los estilos de vida, que tienen implicaciones directas e indirectas en la educación, los valores culturales y la salud (7). El uso indiscriminado de internet, videojuegos y televisión es actualmente el mayor pasatiempo de los adolescentes, y por consiguiente uno de los factores predisponentes a la inactividad física (8); es así como la población infantily adolescente ha dejado de practicar juegos al aire libre para pasar tiempo prolongado frente a un equipo, de donde resultan enfermedades modernas, como nuevas formas de dependencia adictiva, trastornos psicológicos, sobreesfuerzo, estrés asociado al uso de tecnología, obesidad y sedentarismo (7); tales patrones de conducta pueden persistir a lo largo de la vida y tener efectos deletéreos para la salud en el periodo de la adultez (9).

Estudios demuestran la importancia de generar conductas y acciones sobre la salud de los adolescentes para mantenerla o mejorarla, obteniendo así hábitos de vida saludable, tal como lo define la Organización Mundial de la Salud (OMS), una "forma general de vida basada en la interacción entre las condiciones de vida en un sentido amplio y los patrones individuales de conducta determinados por factores socioculturales y características personales" (10). En la población adolescente se encuentran actitudes y 
prácticas no saludables que aumentan los problemas de salud, como el consumo de alcohol, tabaco y sustancias psicoactivas (SPA), las dietas mal sanas, la inactividad física, entre otros (11); estas conductas se convierten en un factor de riesgo determinante para la aparición y aumento en la morbimortalidad de las enfermedades no transmisibles (ENT), como las cardiovasculares, diabetes, cáncer y respiratorias crónicas, responsables del $75 \%$ de las muertes de la población a nivel mundial y el deterioro en la calidad de vida. (12)

En Colombia el Ministerio de Salud y Protección Social (MSPS), a través de la Subdirección de Enfermedades no Transmisibles (SENT) ha implementado la estrategia 4x 4 (13), que orienta sobre la prevención y control de tales enfermedades, y se enfoca en el no consumo de alcohol y sustancias psicoactivas, el control del consumo de tabaco, la alimentación saludable, la promoción de la actividad física; junto con distintas estrategias de comunicación, en cuya población objetivo se encuentran los adolescentes de los entornos escolares.

La intervención sobre los entornos escolares permite avanzar sobre entornos saludables, es decir, escenarios que favorecen entre otras cosas el desarrollo humano sustentable y sostenible; el bienestar individual y colectivo, la salud integral, escenarios que además ofrecen protección, seguridad, confianza para la vida, convivencia de las personas y colectivos; adicional a la gestión, el ejercicio de los derechos y mejores condiciones y calidad de vida (14).

La promoción de los estilos de vida saludable (EVS) en el entorno escolar cuenta, con una amplia normatividad. A nivel nacional, los estilos de vida saludable en los adolescentes están respaldados por la Constitución Política, el Plan de Desarrollo, el Código de Infancia y Adolescencia, la Ley 1438 y el Decreto 1860. La Constitución Política de Colombia es explícita en los derechos de los niños y adolescentes en donde se garantiza el derecho a la salud, el deporte, la alimentación, entre otros, lo cual muestra al sistema de salud como uno de los garantes de estos derechos (15), todos en armonía para brindar mayor probabilidad de disminuir la pérdida de años de vida evitable por condiciones no transmisibles (16).

La intervención sobre los EVS tiene como finalidad disminuir el grado de exposición de las personas a los factores de riesgo modificables, por lo que a nivel específico existe una normatividad vigente para el consumo de tabaco, sustancias psicoactivas, dieta malsana, inactividad física y uso nocivo del alcohol.

Respecto al tabaco, se tiene el Convenio Marco de la OMS para el Control del Tabaco (17), la Ley 1335 (18) que estipula las políticas públicas para la prevención de su consumo en ambientes escolares y el Plan Decenal de Salud Pública (19) que plantea como meta posponer la edad de inicio de consumo de tabaco y alcohol en los adolescentes por encima de los 14 años; en relación con el alcohol, la estrategia $4 \times 4$ busca impactar en este factor, la Ley 30 de 1986 (20) y el Decreto 120 de 2010 (21) buscan controlar el consumo de alcohol al interior de los establecimientos educativos; en cuanto a las sustancias psicoactivas (SPA), la Ley 1566 (22) responsabiliza a las entidades 
administradoras de planes de beneficios $y$ las entidades territoriales en el control de abuso y adicción de estas sustancias; acerca de la salud sexual y reproductiva, la Ley 115 de 1994 (23), determina que el proyecto educativo institucional (PEI) debe contemplar acciones pedagógicas relacionadas con esta área; respecto a la dieta y la actividad física, la Ley 1355 (24) contempla la promoción de actividad física, seguridad alimentaria y nutricional.

A pesar de la amplia normatividad que rodean la intervención sobre los EVS, Pinzón y Vernaza (25) afirman que el departamento del Cauca presenta una alta vulnerabilidad social y económica, lo que probablemente aumenta la exposición a distintos factores de riesgo, como lo es el consumo de sustancias psicoactivas, tabaco, el uso nocivo del alcohol, la inactividad física y la dieta malsana. El propósito de la investigación es determinar los estilos de vida de los entornos escolares, sus conocimientos, actitudes y prácticas hacia su salud, en los estudiantes de educación media vocacional (grados noveno, decimo, once), en seis instituciones educativas públicas y privadas de la ciudad de Popayán.

\section{Materiales y métodos}

Se realizó un estudio de diseño descriptivo, observacional de corte transversal, en seis colegios de la ciudad de Popayán, donde se implementó una estrategia de comunicación en salud en 300 estudiantes del grado noveno y once. La muestra se seleccionó mediante un muestreo probabilístico aleatorio simple, calculada con una precisión del $5 \%$, nivel de confianza del $95 \%$ y una proporción esperada del $20 \%$, donde se seleccionaron 135 estudiantes de instituciones educativas públicas y privadas, para conocer sus actitudes, prácticas y estilos de vida. Se estableció como criterios de inclusión estar matriculado como estudiante de educación media vocacional, la participación voluntaria y el diligenciamiento completo del cuestionario.

Para la recolección de la información, se aplicó un instrumento tipo encuesta de 33 ítems para el análisis de factores sociodemográficos, actitudes, prácticas y hábitos de vida de los adolescentes relacionados con actividad física, dieta, consumo de sustancias psicoactivas, alcohol y tabaco; preguntas tomadas del cuestionario Steps de la OMS. Este se aplicó de forma individual en el aula de clase, sin la presencia de profesores, ni directivos; se recogió protegiendo el anonimato, sin identificación, nombres, números, ni códigos.

Las consideraciones éticas se basaron según Resolución 8430 de 1993 de investigación científica en Colombia, catalogada como un estudio sin riesgo; la participación fue libre y voluntaria, se contó con la aprobación de los directivos de las instituciones educativas y se firmó el formato de consentimiento informado dando la posibilidad de rehusar o abandonar el estudio en cualquier momento sin ningún prejuicio.

Para el análisis estadístico se establecieron como variables dependientes las relacionadas con las actitudes, prácticas y estilos de vida de los adolescentes, y como independientes el género y el grado escolar. En el procesamiento de datos se utilizó el programa de análisis estadístico SPSS v15, para la descripción 
de variables se utilizaron frecuencias, porcentajes, medidas de tendencia central como la media y la desviación estándar y se realizó un análisis bivariado para determinar la asociación de las variables con chi-cuadrado y Odds ratio para observar la probabilidad de riesgo.

\section{Resultados}

La muestra estuvo constituida por 135 estudiantes de 6 instituciones de educación media vocacional de carácter público y privado de la ciudad de Popayán; pertenecen al grado noveno $(51,9 \%)$, al grado once $(47,4 \%)$; corresponden al género femenino $(50,4 \%)$ y al masculino (49,6\%); el rango de edades estuvo entre 13 y 19 años, con una media de 15,8 y $\mathrm{s} \pm 1,21 ; \mathrm{y}$ pertenecen a los estratos uno, dos y tres $(84,3 \%)$.

En cuanto al consumo de tabaco, los estudiantes manifestaron haber fumado (38,5\%); consumido tabaco en los últimos 30 días (10,4\%); y tener conocimiento de los efectos negativos de su consumo (83,7\%). En general, se encontró una edad de inicio mínima de 8 y máxima de 18 años ( $\mathrm{x}=$ $13,9 ; s \pm 1,7)$, se observó que en el género femenino la edad de inicio mínima fue de 12 y la máxima de 18 años $(\mathrm{x}=14,4 ; \mathrm{s} \pm$ $1,5)$, mientras que en los hombres la edad de inicio mínima fue de 8 y máxima de 16 años $(x=13,5 ; s \pm 1,8)$ (tabla 1$)$.

En lo que se refiere al consumo de alcohol, los estudiantes manifestaron haberlo consumido (88,9\%), ingerido en los últimos 30 días $(37 \%)$ y conocer sus efectos negativos $(86,7 \%)$. Se encontró una edad de inicio mínima de 9 y una máxima de 17 años ( $\mathrm{x}$ $=13,4 ; \mathrm{s} \pm 1,7)$, se observó que en el género femenino como en el masculino la edad de inicio mínima fue de 9 y máxima de 17 años (mujeres: $\mathrm{x}=13,6 \%$; \pm 1,7); (hombres: $\mathrm{x}=13,2 \%$; $\mathrm{s} \pm 1,8)$ (tabla 2).

Respecto al consumo de sustancias psicoactivas (SPA), los adolescentes manifestaron haber consumido algún tipo de sustancia (29,6\%) y conocer los efectos negativos de su consumo (82,2\%). En general, se encontró una edad de inicio mínima de 10 y una máxima de 18 años $\left(\mathrm{x}^{-}=14,1 \%\right.$; $s \pm 1,5)$, se observó que en el género femenino la edad de inicio mínima fue de 13 y máxima de 15 años $(x=13,9 \%$; $s \pm 0,9)$; mientras que en los hombres la edad de inicio mínima fue de 10 y máxima de 18 años $\left(\mathrm{x}^{-}=14,2 \%\right.$; $\left.\mathrm{s} \pm 1,7\right)$ (tabla 2$)$.

Al indagar sobre las relaciones sexuales, los estudiantes manifestaron haber iniciado su vida sexual (59,3\%). Se encontró una edad de inicio mínima de 10 y una máxima de 17 años $(x=14,3 ; s \pm 1,4)$, se observó que en el género femenino la edad de inicio mínima fue de 13 y máxima de 17 años $\left(\mathrm{x}^{-}=14,9\right.$; $\mathrm{s} \pm 0,9)$; mientras que en los hombres su edad de inicio mínima fue de 10 y máxima de 17 años $(\mathrm{x}=13,8 \%$; $\mathrm{s} \pm 1,6)$; en cuanto al conocimiento de los estudiantes sobre las enfermedades de transmisión sexual(ETS), manifestaron no tener conocimiento sobre el tema $(5,9 \%)$ (tabla 2$)$.

Sobre el conocimiento de los métodos anticonceptivos, se encontró que los adolescentes conocían el uso del condón $(97,8 \%)$, las pastillas anticonceptivas (77\%), la pila $(65,9 \%)$, la ligadura de tropas $(48,9 \%)$, la vasectomía $(42,4 \%)$, la inyección hormonal (34,8\%), el dispositivo intrauterino (20\%), mientras que el ritmo era el método menos conocido (11,1\%). 
Tabla 1. Características, actitudes y prácticas de los adolescentes de secundaria Popayán

\begin{tabular}{|c|c|c|c|c|c|}
\hline & n..$^{\circ}$ & $\%$ & & n. $0^{\circ}$ & $\%$ \\
\hline \multicolumn{3}{|l|}{ Género } & \multicolumn{3}{|l|}{ Ha fumado } \\
\hline Femenino & 68 & 50,4 & Sí & 52 & 38,5 \\
\hline Masculino & 67 & 49,6 & No & 83 & 61,5 \\
\hline \multicolumn{3}{|l|}{ Grado escolar } & \multicolumn{3}{|c|}{ Consumido tabaco en los últimos $\mathbf{3 0}$ días } \\
\hline Noveno & 70 & 51,9 & Sí & 14 & 10,4 \\
\hline Once & 65 & 48,1 & No & 121 & 89,6 \\
\hline \multicolumn{3}{|c|}{ Estrato socioeconómico } & \multicolumn{3}{|c|}{ Conoce los efectos negativos del tabaco } \\
\hline 1 a 3 & 114 & 84,3 & Sí & 113 & 83,7 \\
\hline 4 a 6 & 21 & 15,7 & No & 22 & 16,3 \\
\hline \multicolumn{3}{|c|}{ Actividad en tiempo libre } & \multicolumn{3}{|c|}{ Ha consumido alcohol } \\
\hline Ejercicio & 40 & 29,6 & Sí & 120 & 88,9 \\
\hline PC, celular, tablet & 37 & 27,4 & No & 15 & 11,1 \\
\hline Ver televisión & 8 & 5,9 & \multicolumn{3}{|c|}{ Consumido alcohol en los últimos 30 días } \\
\hline Oír música & 45 & 33,3 & Sí & 50 & 37 \\
\hline \multicolumn{3}{|c|}{ Realiza actividad Física } & No & 85 & 63 \\
\hline Sí & 118 & 87,4 & \multicolumn{3}{|c|}{ Conoce los efectos negativos del alcohol } \\
\hline No & 17 & 12,6 & Sí & 117 & 86,7 \\
\hline \multicolumn{3}{|l|}{ Ha consumido SPA } & No & 18 & 13,3 \\
\hline Sí & 40 & 29,6 & \multicolumn{3}{|l|}{ Conoce las ETS } \\
\hline No & 95 & 70,4 & Sí & 127 & 94,1 \\
\hline \multicolumn{3}{|c|}{ Conoce los efectos negativos de las SPA } & No & 8 & 5,9 \\
\hline Sí & 111 & 82,2 & & & \\
\hline No & 24 & 17,2 & & & \\
\hline Total & 135 & $100 \%$ & Total & 135 & $100 \%$ \\
\hline
\end{tabular}

Fuente: Elaboración propia con base en los resultados.

En relación con la actividad física, los estudiantes realizan esta actividad $(87,4 \%)$, con una frecuencia de 1 a 2 veces por semana (45,9\%), de 3 a 4 veces $(41,5 \%)$ y de 5 a 7 veces por semana $(11,1 \%)$; el estudio además indicó que las mujeres $(55,9)$ son más sedentarias que los hombres $(35,8)$.

Al indagar sobre las actividades que realizan en su tiempo libre en un día común, se encontró quelos adolescentes escucharon música $(33,3 \%)$, realizaron ejercicio $(29,6 \%)$, usaron aparatos electrónicos como PC, tablet o celular $(27,4 \%)$ y vieron televisión (5,9\%).

Al indagar sobre la dieta, se evidenció que los estudiantes consumen frutas de 1 a 2 veces por semana $(43,7 \%)$, de 3 a 4 veces $(39,3 \%)$ y de 5 a 7 veces $(13,3 \%)$, y no consumen ninguna fruta $(3,7 \%)$. Respecto a las verduras, las consumen de 1 a 2 veces por semana $(36,3 \%)$, de 3 a 4 veces (40\%) y de 5 a 7 veces (20\%), y manifiestan no consumirlas (3\%). 
Tabla 2. Edad de inicio prácticas no saludables en adolescentes de secundaria

\begin{tabular}{|c|c|c|c|c|c|}
\hline & & & & & \\
\hline & \multicolumn{5}{|c|}{ Rango edad de inicio } \\
\hline & $\%$ & Min & Max & $x-$ & $\mathbf{S} \pm$ \\
\hline Consumo de tabaco & 38,5 & 8 & 18 & 13,9 & 1,7 \\
\hline Mujeres & 16,2 & 12 & 18 & 14,4 & 1,5 \\
\hline Hombres & 22,2 & 8 & 16 & 13,5 & 1,8 \\
\hline Consumo alcohol & 88,9 & 9 & 17 & 13,4 & 1,7 \\
\hline Mujeres & 44,4 & 9 & 17 & 13,6 & 1,7 \\
\hline $\begin{array}{c}\text { Hombres } \\
\text { Consumo SPA } \\
\text { Mujeres } \\
\text { Hombres } \\
\text { Inicio relaciones sexuales } \\
\text { Mujeres } \\
\text { Hombres }\end{array}$ & $\begin{array}{c}44,4 \\
29,6 \\
8,1 \\
21,4 \\
59,3 \\
25,9 \\
33,3\end{array}$ & $\begin{array}{c}9 \\
10 \\
13 \\
10 \\
10 \\
13 \\
10\end{array}$ & $\begin{array}{l}17 \\
18 \\
15 \\
18 \\
17 \\
17 \\
17\end{array}$ & $\begin{array}{l}13,2 \\
14,1 \\
13,9 \\
14,2 \\
14,3 \\
14,9 \\
13,8\end{array}$ & $\begin{array}{l}1,8 \\
1,5 \\
0,9 \\
1,7 \\
1,4 \\
0,9 \\
1,6\end{array}$ \\
\hline
\end{tabular}

Fuente: Elaboración propia con base en los resultados.

En relación con el consumo de comidas rápidas, los estudiantes manifiestan consumirlas de 1 a 2 veces por semana $(72,6 \%)$, de 3 a 4 veces $(17,8 \%)$, de 5 a 7 veces $(7,4 \%)$ y otros afirman no consumir comidas rápidas $(2,2 \%)$. En cuanto al elemento utilizado para freír alimentos en casa, se encontró el aceite $(90,4 \%)$ y la manteca o mantequilla $(5,2 \%)$ como los más usados.

En el análisis bivariado de las actitudes, prácticas y estilos de vida de los adolescentes, se identificó una relación estadísticamente significativa entre el género y la actividad física $(\mathrm{p}=0,06)$; género y si había consumido algún tipo de SPA $(\mathrm{p}=0,01)$; grado escolar y si había iniciado relaciones sexuales $(p=0,03)$, y grado escolar con consumo de alcohol en los últimos 30 días. En el análisis multivariado se observó que ser hombre es un factor protector para la realización de actividad física $(\mathrm{OR}=0,242 \mathrm{IC} 95 \%=0,08-0,70)$; pero es un factor que aumenta el riesgo para consumir sustancias psicoactivas $(\mathrm{OR}=1,813 \mathrm{IC} 95 \%=1,32-2,47)$. Se encontraron 2 determinantes protectores, el pertenecer al grado noveno en relación con haber iniciado una vida sexual activa $(\mathrm{OR}=0,34 \mathrm{IC} 95 \%=0,16-0,70)$ y el haber consumido alcohol en los últimos 30 días $(\mathrm{OR}=0,43 \mathrm{IC} 95 \%=0,20-0,88)$ (tabla 3$)$.

\section{Discusión}

El estudio pone de manifiesto los estilos de vida de los entornos escolares, sus conocimientos, actitudes y prácticas hacia su salud, en los estudiantes de educación media vocacional, en seis instituciones educativas públicas y privadas de la ciudad de Popayán. 
Tabla 3. Análisis bivariado

\begin{tabular}{|c|c|c|c|c|c|c|}
\hline & \multicolumn{2}{|c|}{ Género } & \multirow[b]{2}{*}{$\mathbf{X} 2$} & \multirow[b]{2}{*}{ OR } & \multirow[b]{2}{*}{ IC $95 \%$} & \multirow[b]{2}{*}{$\mathbf{p}$} \\
\hline & Femenino & Masculino & & & & \\
\hline \multicolumn{7}{|c|}{ Actividad física } \\
\hline Sí & 51 & 62 & 7,609 & 0,242 & $0,084-0,701$ & 0,06 \\
\hline No & 17 & 5 & & & & \\
\hline \multicolumn{7}{|c|}{ Ha consumido SPA } \\
\hline Sí & 11 & 29 & 11,89 & 1,813 & $1,327-2,475$ & 0,01 \\
\hline No & 57 & 38 & & & & \\
\hline \multicolumn{7}{|c|}{ Grado escolar } \\
\hline & Noveno & Once & & & & \\
\hline \multicolumn{7}{|c|}{ Inició vida sexual } \\
\hline Sí & 33 & 47 & 8,841 & 0,342 & $0,167-0,700$ & 0,03 \\
\hline No & 37 & 18 & & & & \\
\hline \multicolumn{7}{|c|}{ Alcohol últimos 30 días } \\
\hline Sí & 18 & 29 & 5,30 & 0,430 & $0,208-0,888$ & 0,02 \\
\hline No & 52 & 36 & & & & \\
\hline
\end{tabular}

Fuente: Elaboración propia con base en los resultados.

Respecto al consumo de tabaco en la población estudio, el $38,5 \%$ ha fumado alguna vez, situación similar se evidenció en la investigación realizada en el estado de Nuevo León-México, donde se constató una prevalencia de tabaquismo de 30,5\% (26); al mismo tiempo, en Yucatán y Coahuila-México, se afirma que el tabaco fue la principal droga de inicio con el 35,1\% (27), situación probablemente asociada al aumento de consumo de tabaco con la edad de los estudiantes y la vulnerabilidad a estas sustancias al encontrarse en pleno desarrollo (27).

La edad de inicio para consumo de tabaco es cada vez más temprana, en el presente estudio el $26,6 \%$ de los estudiantes inició su consumo entre los 8 y 18 años de edad. deraron consumidores de cigarrillo, con una edad promedio de inicio de consumo entre 16 y 17 años; de modo similar, López et al. (26) afirman que la edad promedio de inicio del consumo de tabaco es a los 14 años. Martínez (28) afirma que en las últimas décadas se ha observado el inicio del consumo de tabaco a edades muy tempranas. Esto pone de manifiesto que la meta propuesta por el Plan Decenal de Salud Pública (PDSP) (2012-2021) sobre aumentar la edad de inicio a los 14 años no ha logrado un impacto suficiente. (19).

En relación con las bebidas alicoradas, el estudio pone de manifiesto que el $37 \%$ de los estudiantes consumió alcohol en los últimos 30 días, situación similar a la encontrada por Pérez et al. (29) realizada en nueve ciudades de Colombia, donde el 36\% afirmó haber consumido alcohol 
en el último mes, indicando que los adolescentes son consumidores habituales de este tipo de bebidas.

El estudio encontró que la edad promedio de inicio para el consumo de alcohol fueron los 13 años, similar a los resultados hallados por Pérez et al. (29), donde la edad de inicio estaba alrededor de los 12 años, señalando además la unión familiar y el seguimiento tradicional de la cultura como los valores que promueven el consumo de alcohol a edades tempranas, por lo que la responsabilidad del uso de bebidas embriagantes recae sobre la familia y no sobre el adolescente. Adicional a esto, Pérez et al. (29) encontraron que el 14\% de los participantes iniciaron el consumo de alcohol antes de los 10 años, con una mayor tendencia en los hombres que en las mujeres, sin embargo, el presente estudio identificó una tendencia similar tanto para hombres como para mujeres.

Simultáneamente, en un estudio realizado con estudiantes universitarios de Medellín, Castaño et al. (30) encontraron que la edad de inicio promedio fue de 14 años, resultados que no se alejan del presente estudio, los autores además indican que el consumo de alcohol en esta población se debió a las expectativas que tuvieron los adolescentes sobre el alcohol, como un elemento reductor de la tensión psicológica, desinhibidor y especialmente facilitador de la interacción social. Y Gaete et al. (31), basados en los hallazgos de distintos estudios, sustentan que entre más temprana sea la edad de inicio del consumo de alcohol, el adolescente tiene un mayor riesgo de bajo rendimiento académico con mayor probabilidad de desarrollar en la edad adulta consumo excesivo de alcohol, tendencia al suicidio, violencia y problemas conductuales.

Acerca de las sustancias psicoactivas, el estudio indicó que el 29,6\% de los participantes afirmaron haberlas consumido por lo menos una vez en su vida, a diferencia de lo encontrado por Londoño y Álvarez (32), donde el consumo fue de $65,5 \%$ de su población estudio. Por su parte, Villatoro et al. (33), expresan que el $17,2 \%$ de los estudiantes de México las han consumido; además, se afirma que los departamentos de Caldas, Antioquia, Risaralda, Quindío, y Bogotá D.C. presentan los niveles más altos de uso de alguna de estas sustancia (34).

Los anteriores porcentajes son superiores frente a los resultados obtenidos en el Estudio Nacional de Consumo de Sustancias Psicoactivas (35), donde se encontró que el 13\% de las personas encuestadas han usado por lo menos una vez en la vida alguna sustancia ilícita; este mismo estudio pone de manifiesto que "las diferencias del consumo de estas sustancias por sexo son considerables, siendo el consumo de los varones tres veces superior al consumo de las mujeres" (35).

La edad de inicio para el consumo de SPA es una de las situaciones más preocupantes para el Estado colombiano, en el estudio la edad promedio de inicio se encuentra en los 14 años de edad, al mismo tiempo, Villatoro et al. (33) manifiestan que la edad promedio para el consumo de estas sustancias es de 13 años. De modo similar, Nabalon y Ruiz (36) encontraron que la edad de inicio para el consumo de cannabis es de 14 años de edad, siendo esta la sustancia ilícita más consumida en el mundo. Por otro lado, la edad para las 
drogas duras como cocaína, speed y éxtasis se sitúa en los 15 años de edad. Cabe señalar que "a pesar de la gran diferencia detectada entre hombres y mujeres en cuanto a la magnitud del uso de sustancias ilícitas, no se observan diferencias en términos de la edad de inicio" (35).

El género y el consumo de sustancias psicoactivas presentó una relación estadísticamente significativa, donde ser hombre es un factor de riesgo para el consumo de estas sustancias. Esta idea se complementa con Caravaca et al. (37), quienes afirman que el género femenino presentó menor probabilidad de consumir marihuana, cocaína y anfetaminas; y en la guía de género y drogas (38) se observa la disminución del consumo en el género femenino y puede ser el resultado dela percepción que tienen las mujeres del riesgo asociado a la utilización frecuente de estas sustancias.

En cuanto a la actividad sexual, los resultados muestran que la edad de inicio de las relaciones sexuales en los adolescentes ocurre a temprana edad (14 años). Holguin et al. (39) encontraron resultados similares en los estudiantes de colegios públicos de Tuluá-Colombia (15 años), los cuales coinciden además con la investigación sobre prácticas sexuales de los adolescentes escolarizados de Socha-Boyacá (15 años) (40). Se debe agregar que Holguin et al. (39) enfatizan en las consecuencias de una sexualidad precoz y una maternidad en la adolescencia, en la salud, el desarrollo educativo y personal, así como la disminución de población con potencial de productividad para la sociedad.

Por otro lado, los resultados indican que los hombres presentan edades más tem- pranas de inicio de la actividad sexual con edades tan inferiores como los 10 años. Así mismo, Holguin et al. (39) mostraron que, en comparación con las mujeres, los hombres inician su vida sexual de forma más temprana. Es importante destacar que distintos estudios han mostrado que entre los determinantes individuales más importantes para el inicio tempano de la actividad sexual se describen el sexo masculino, el consumo de alcohol, tabaco y drogas. Por otra parte, Cabrera et al. (40) citan a Campo Arias para expresar que postergar el inicio de las relaciones sexuales genera importantes implicaciones en la salud pública, ya que se reducen los embarazos no deseados, las muertes por abortos intencionados y las enfermedades de transmisión sexual, entre otros.

Respecto a los métodos anticonceptivos, el condón fue el más conocido $(97,8 \%)$ seguido de las pastillas anticonceptivas (77\%), guardando estrecha relación con el estudio de Sánchez et al., (41) realizado en México, donde gran parte de los encuestados tenían conocimiento del condón (100\%) y de los métodos hormonales por vía oral (87,5\%); sin embargo, métodos como la inyección o el dispositivo intrauterino son poco conocidos, lo que evidencia la falta de orientación en esta área. A este respecto, Sánchez et al. manifiestan que "el problema de salud sexual en los adolescentes no es el inicio de la vida sexual a una edad temprana, sino la carencia de orientación y conocimiento adecuado para una conducta sexual responsable" (41).

En relación con el tiempo que dedican los adolescentes para realizar actividad física (A.F), la OMS recomienda efectuar movimientos corporales que conduzcan a 
un gasto energético (3), donde los grupos etarios entre 5 y 17 años deben dedicarle por lo menos tres días a la semana (42). En este estudio, se determinó que un alto porcentaje de adolescentes no presentaba un nivel suficiente de A.F. (45,9\%), resultado similar al presentado en México D.F., donde el 66,3\% de los encuestados presentan un nivel de A. F. menor al recomendado (9); a su vez, los resultados de la Encuesta Nacional de Situación Nutricional (ENSIN)-2010, en Colombia evidencian que el $74 \%$ de la población no cumple con el mínimo de actividad física. Como resultado, la OMS indica que estas personas "presentan un riesgo de mortalidad, entre un $20 \%$ y un $30 \%$ superior al de aquellas que son lo suficientemente activas" (3).

Otros estudios muestran que las mujeres son menos activas que los hombres; Yáñez et al. (43) evidenciaron en su investigación que el 23,88\% de las mujeres incumplían las recomendaciones de la OMS, frente a un $28,46 \%$ en el caso de los varones; González y Portolés (44) muestran 53,2\% y $32,8 \%$, respectivamente, y documentan la disminución de la A.F., como resultado del incremento del tiempo que los adolescentes pasan frente al computador o televisor y que favorecen las conductas sedentarias, $(29,6 \%)$; por otra parte, según González et al. (45), el uso del tiempo libre en actividades deportivas está asociado significativamente a una menor frecuencia de consumo de alcohol, tabaco y drogas ilícitas en estudiantes de secundaria, razón por la cual debe ser la base de las estrategias de prevención de consumo de drogas en adolescentes.

En cuanto a la relación entre la actividad física y el género, se encontró que ser hombre es un factor protector para la realización de esta. De modo similar, Lavielli et al. (9) afirman que el sedentarismo se asocia al género, donde las mujeres tienen mayor predisposición a él y que puede estar asociado a los estereotipos y juicios socioculturales respecto a la participación de las mujeres en algún tipo de actividad física tales, como afectación de la feminidad, alteración del ciclo menstrual, daños en los órganos reproductores, y que además pueda generar consecuencias negativas en la fertilidad. (9)

Respecto a la dieta, la OMS recomienda "consumir más de 400 gramos de frutas y verduras al día para mejorar la salud general", sin embargo, el consumo de estos alimentos por parte de los adolescentes de forma diaria no es alto, por el contrario, un importante porcentaje de los estudiantes los consume solo de 1 a 2 veces por semana. Este consumo insuficiente de frutas y verduras, según la OMS, es uno de los 10 factores principales de riesgo de mortalidad a escala mundial (46). Resultados similares se obtuvieron en la Universidad Nacional Mayor de San Marcos, en la cual solo el 7,4\% de la muestra consumió 5 o más porciones al día (47), a su vez el consumo promedio de frutas $(1,81 \%)$ y verduras $(1,23 \%)$ por parte de sus estudiantes fue bajo y también en Argentina, donde solo el 17,6\% de los adolescentes consumió más de 5 porciones diarias de frutas y verduras y el 6,8\% consumió alimentos procesados como las comidas rápidas (48).

\section{Conclusiones}

Las causas de sus hábitos no saludables se ven influenciadas por su vulnerabilidad 
y círculo social, así como también por el uso de tecnología y el ritmo de la vida cotidiana, influyen directamente sobre los determinantes de salud de esta población.

El consumo de alcohol, tabaco y sustancias psicoactivas no presentó relación significativa con la estratificación social ni con el grado escolar, dando a entender que el factor de riesgo está presente en todos los adolescentes de educación media vocacional.

El estudio pone en evidencia que los estilos de vida, actitudes y prácticas poco saludables en los entornos escolares, como el consumo de alcohol, tabaco, SPA, dietas malsanas, entre otros aspectos, trae como

\section{Referencias}

1. Juárez C, Márquez M, Salgado N, Pelcastre B, Ruelas M, Reyes H. La desigualdad en salud de grupos vulnerables de México: adultos mayores, indígenas y migrantes. Rev Panam Salud Publica [Internet]. 2014 [citado 20 Jun 2017]; 35(4):284-290. Disponible en: http://iris.paho.org/xmlui/ bitstream/handle/123456789/8166/08.pdf? sequence $=1$ \&isAllowed $=y$

2. García J. Concepto de Vulnerabilidad Psicosocial en el Ámbito de la Salud y las adicciones. Rev Health and Addictions [Internet]. 2015 [citado 20 June 2017]; 15(1):5-14. Disponible en: http://www.redalyc.org/html/839/83938758001/

3. Actividad física [Internet]. Organización Mundial de la Salud. 2017 [actualizado 6 Feb 2017; citado 27 Jun 2017]. Disponible en: http://www.who.int/mediacentre/ factsheets/fs385/es/

4. Medina Ó, Rubio L. Consumo de sustancias psicoactivas (SPA) en adolescen- consecuencia un aumento significativo en el riesgo de padecer enfermedades crónicas no trasmisibles (ECNT) a edades tempranas.

Los resultados obtenidos en este estudio muestran la necesidad de establecer e implementar políticas públicas encaminadas a la promoción de hábitos de vida saludable y prevención de consumo de sustancias nocivas en los adolescentes. Resaltando que se deben dirigir a la población en general, pero de manera prioritaria a menores de edad y sus acudientes.

Conflicto de intereses: Los autores declaran no tener ningún conflicto de intereses.

tes farmacodependientes de una fundación de rehabilitación colombiana. Estudio descriptivo. Rev. Colomb. Psiquiatr. [Internet]. 2012 [citado 20 Jun 2013]; 41(3):550561. Disponible en: http://www.scielo. org.co/scielo.php?script=sci_arttext\&pi$d=S 0034-74502012000300007 \&$ Ing $=$ en.

5. El alcoholismo. 9. Influencia de las Amistades. [Internet]. Bogotá: El alcoholismo; 2013 [actualizado 30 Nov 2013; citado 20 Jun 2017]. Disponible en: https://alcoholismo222.wordpress.com/2013/11/30/influencia-de-las-amistades-2/

6. Ruiz V, Londoño N. Caracterización psicosocial de la población consumidora de SPA, en proceso de rehabilitación vinculada a la comunidad terapéutica Faro del departamento del Quindío. Revi Elect Psicolog Soc Poiés [Internet]. 2010 [citado 20 Jun 2017]; 19(1):1-8. Disponible en: http://www.funlam.edu.co/revistas/index. php/poiesis/article/view/109/88 
7. Quintero J, Munévar R, Munévar Q. Nuevas tecnologías, nuevas enfermedades en los entornos educativos. Rev Hac Promoc Sal [Internet]. 2015 [citado 20 Jun 2017];20(2):13-26. Disponible en: http://www.scielo.org.co/pdf/hpsal/v20n2/ v20n2a02.pdf

8. Collipal E, Godoy M. Representaciones sociales de la obesidad en jóvenes preuniversitarios y universitarios. Internat Jour Morph [Internet]. 2015 [citado 20 Jun 2017]; 33(3):877-882. Disponible en: http://www.scielo.cl/pdf/ijmorphol/v33n3/ art12.pdf

9. Lavielle $P$, Pineda V, Jáuregui $O$, Castillo M. Actividad física y sedentarismo: Determinantes sociodemográficos, familiares y su impacto en la salud del adolescente. Rev Salud Públic [Internet]. 2014 [citado 20 Jun 2017]; 16(2):161-172. Disponible en: http://www.scielosp.org/pdf/ rsap/v16n2/v16n2a01.pdf

10. Cerón S. Editorial. Rev Univ. Salud. [Internet]. 2012 [citado 20 Jun 2017]; 14(2):115-115. Disponible en: http://www. scielo.org.co/scielo.php?script=sci arttext\&pid=S0124-71072012000200001\&lng=en

11. Sánchez M, De Luna E. Hábitos de vida saludable en la población universitaria. Rev Nutr Hosp [Internet]. 2015 [citado 20 June 2017]; 31(5):1910-1919. Disponible en: http://www.redalyc.org/articulo. oa? $\mathrm{id}=309238514003$.

12. Enfermedades no transmisibles [Internet]. Organización Mundial de la Salud. 2017 [actualizado 10 Jun 2017; citado 20 June 2017]. Disponible en: http://www. who.int/mediacentre/factsheets/fs355/es/

13. Organización Mundial de la Salud. Prevención y control de las enfermedades no transmisibles: aplicación de la estrategia mundial [Internet]. Ginebra: Consejo Ejecutivo; 2008 [citado 6 Feb 2017]. Disponible en: http://www.who.int/gard/ publications/B122_9-sp.pdf
14. Colombia M. Páginas-Entornos Saludables [Internet]. Minsalud.gov.co. 2017 [actualizado 10 Feb 2017; citado 25 June 2017]. Disponible en: https://www.minsalud.gov.co/proteccionsocial/Paginas/EntornosSaludables.aspx

15. Congreso de Colombia. Consulta de la Norma: [Internet]. Alcaldiabogota.gov. co. 2017 [actualizado 10 Feb 2000; citado 6 Feb 2017]. Disponible en: http://www. alcaldiabogota.gov.co/sisjur/normas/Norma1.jsp?i=4125

16. Departamento Nacional de Planeación. Movilidad Social. En: Departamento Nacional de Planeación, ed. por. Plan Nacional de Desarrollo 2014-2018 [Internet]. 1st ed. Bogotá: Grupo de Comunicaciones y Relaciones Públicas; 2015 [citado 6 Feb 2017]. p. 310-311. Disponible en: https://colaboracion.dnp.gov.co/CDT/PND/PND\%2020142018\%20Tomo\%201\%20internet.pdf

17. Organización Mundial de la Salud. Convenio Marco de la OMS para el control del tabaco [Internet]. 2nd ed. Ginebra: OMS; 2005 [citado 6 Jun 2017]. Disponible en: http://www.who.int/fctc/text_download/es/

18. Congreso de Colombia. Consulta de la Norma: Ley 1335 [Internet]. Alcaldiabogota.gov.co. 2009 [actualizado 21 Jun 2009; citado 20 Jun 2017]. Disponible en: http://www.alcaldiabogota.gov.co/sisjur/ normas/Norma1.jsp?i=36878

19. Ministerio de Salud y Protección Social. Plan Decenal de Salud Pública 20122021: La salud en Colombia la construyes tú [Internet]. 1st ed. Bogotá: Grupo de Comunicaciones; 2013 [citado 6 Feb2017]. Disponible en: https://www.minsalud.gov. co/Documentos $\% 20 y \% 20$ Publicaciones/ Plan\%20Decenal\%20-\%20Documento $\% 20$ en $\% 20$ consulta $\% 20$ para $\% 20$ aprobaci\%C3\%B3n.pdf

20. Congreso de Colombia. Consulta de la Norma: [Internet]. Alcaldiabogota.gov. 
co. 2017 [actualizado 20 Jun 2009; citado 6 Feb 2017]. Disponible en: http://www. alcaldiabogota.gov.co/sisjur/normas/Norma1.jsp?i=2774

21. Congreso de Colombia. Consulta de la Norma: Ley 30 [Internet]. Alcaldiabogota.gov.co. 2017 [actualizado 21 Ene 2010; citado 25 Jun 2017]. Disponible en: http://www.alcaldiabogota.gov.co/sisjur/ normas/Norma1.jsp?i=38680

22. Congreso de Colombia. Consulta de la Norma: Ley 1566 [Internet]. Alcaldiabogota.gov.co. 2012 [actualizado 31 Jul 2012; citado 20 June 2017]. Disponible en: http://www.alcaldiabogota.gov.co/sisjur/normas/Norma1.jsp?i=48678

23. Congreso de Colombia. Consulta de la Norma: Ley 115 DE 1994 [Internet]. Alcaldiabogota.gov.co. 2000 [actualizado 20 Feb 2000; citado 6 Feb 2017]. Disponible en: http://www.alcaldiabogota.gov.co/ sisjur/normas/Norma1.jsp?i=292

24. Congreso de Colombia. Consulta de la Norma: Ley 1355 de 2009 [Internet]. Alcaldiabogota.gov.co. 2009 [actualizado 23 Mar 2009; citado 6 Feb 2017]. Disponible en: http://www.alcaldiabogota.gov.co/ sisjur/normas/Norma1.jsp?i=37604

25. Vernaza P, Pinzón M. Comportamientos de salud y estilos de vida en adolescentes de tres colegios del municipio de Popayán. Rev Sal Public [Internet]. 2012 [citado 6 Feb 2017];14(6):946-955. Disponible en: http://revistas.unal.edu.co/index. php/revsaludpublica/article/view/30179

26. López M, Alonso M, Méndez M, Armendáriz N. Descripción del consumo de tabaco y alcohol en adolescentes de complementos urbanos del estado de Nuevo León, México. Tobacco And Alcohol Consumption Among Adolescents Of Urban Areas In Nuevo León, México. Health and Addictions Journal/Rev Sal Drog [Internet]. 2016 [citado 20 June 2017]; 16(2):127-134. Disponible en: http://ojs. haaj.org/index.php/haaj/article/view/254
27. Sánchez A, Andueza M, Santana A, Hoil J, Cufarfan J. Características sociodemográficas y perfil de consumo de tabaco y drogas en estudiantes de dos universidades de México. Rev Biomed [Internet]. 2017 [citado 20 Jun 2017];28(1):11-27. Disponible en: http:// new.medigraphic.com/cgi-bin/resumen. cgi?IDARTICULO=70760

28. Martínez-Vispo C, Becoña E. La sensibilidad a la ansiedad y el consumo de tabaco: una revisión. Rev Ansied y Estr. [Internet]. 2016 [citado 20 Jun 2016]; 22 (2-3):118-122. Disponible en: http://www. sciencedirect.com/science/article/pii/ S1134793716300367

29. Pérez A, Mejía J, Reyes M. II Estudio de consumo de alcohol en menores en siete ciudades capitales y dos municipios pequeños de Colombia: 2015 [Internet]. 1st ed. Bogotá; 2015 [citado 6 Feb 2017]. Disponible en: http://nuevosrumbos.org/wp-content/uploads/2015/12/ Consumo-de-Alcohol-en-menores-deedad-en-Colombia-2015.pdf

30. Castaño G, García J, Marzo J. Consumo de alcohol y factores intervinientes en estudiantes universitarios. Rev Cub Sal Public [Internet]. 2014 [citado 20 Jun 2017]; 40(1):47-54. Disponible en: http:// scielo.sld.cu/scielo.php?script=sci_arttext\&pid=S0864-34662014000100006

31. Gaete J, Olivares E, Rojas C, Rengifo $M$, Labbé $N$, Lepe $L$ et al. Consumo de tabaco y alcohol en adolescentes de 10 a 14 años de la ciudad de San Felipe, Chile: prevalencia y factores asociados. Revi Médic Chil [Internet].2016 [citado 20 June 2017]; 144(4):465-475. Disponible en: http:// www.scielo.cl/scielo.php?script=sci_arttext\&pid=S0034-98872016000400007

32. Londoño J, Álvarez M. Consumo de sustancias psicoactivas en auxiliares de enfermería. Rev Cuidart [Internet]. 2017 [citado 20 June 2017];8(2):1591. Disponible en: https://www.revistacuidarte.org/index.php/cuidarte/article/view/378/786 
33. Villatoro J, Medina M, del Campo R, Fregoso D, Bustos M, Resendiz E et al. El consumo de drogas en estudiantes de México: tendencias y magnitud del problema. Salud Mental [Internet]. 2016 [citado 20 June 2017]; 39(4):193-203. Disponible en: http://new.medigraphic.com/cgi-bin/ resumen.cgi?IDARTICULO=67799

34. MinSalud, MinEducación. Resultados del II Estudio de Consumo de Sustancias Psicoactivas en Estudiantes de Secundaria en Colombia [Internet]. 1st ed. Bogotá: MinSalud; 2012 [citado 20 June 2017]. Disponible en: http://www.mineducacion.gov.co/ cvn/1665/articles-303188_recurso_1.pdf

35. MinJusticia, MinSalud, Observatorio de Drogas de Colombia. Estudio Nacional de Consumo de Sustancias Psicoactivas en Colombia-2013 [Internet]. 1st ed. Bogotá; 2013 [citado 6 Feb 2017]. Disponible en: https:// www.unodc.org/documents/colombia/2014/ Julio/Estudio_de_Consumo_UNODC.pdf

36. Nabalon A, Ruiz R. Consumo de sustancias psicoactivas y rendimiento académico. Una investigación en estudiantes de educación secundaria obligatoria. Rev Health and Addictions [Internet]. 2017 [citado 20 June 2017];17(1):45-52. Disponible en: http://ojs.haaj.org/index.php/haaj/ article/view/278/278

37. Morera J, Noh S, Hamilton $\mathrm{H}$, Brands B, Gastaldo D, Wright M. Factores socioculturales y consumo de drogas entre estudiantes universitarios costarricenses. Rev Texto \& Contexto-Enfermagem [Internet]. 2015 [citado 20 June 2017]; 24 (spe):145-153. Disponible en: http://www. scielo.br/pdf/tce/v24nspe/0104-0707-tce24-spe-00145.pdf

38. Sánchez L. Género y droga. Guía Informativa [Internet]. 5th ed. Alicante: Diputación de Alicante. Área de Igualdad y Juventud; 2012 [citado 20 Jun 2017]. Disponible en: http://www.pnsd.msssi.gob. es/profesionales/publicaciones/catalogo/ bibliotecaDigital/publicaciones/pdf/GuiaGenero_Drogas.pdf
39. Holguín $Y$, Mendoza L, Esquivel C, Sánchez R, Daraviña A, Acuña M. Factores asociados al inicio de la actividad sexual en adolescentes de Tuluá, Colombia. Revi Chilen Obstec y Ginec [Internet]. 2013 [citado 20 June 2017];78(3):209219. Disponible en: http://10.4067/s071775262013000300007

40. Cabrera V, Salazar P, Docal M, Aya V, Ardila M, Rivera R. Estilo de vida de los jóvenes y las necesidades de educación sexual [Internet]. Bogotá: Procuraduría General de la Nación-Instituto de Estudios del Ministerio Público; 2013 [citado 6 February 2017]. Disponible en: http:// www.mineducacion.gov.co/cvn/1665/articles-340716_archivo_pdf.pdf

41. Sánchez-Meneses M, Dávila-Mendoza R, Ponce-Rosas E. Conocimiento y uso de métodos anticonceptivos en adolescentes de un centro de salud. Atención Familiar [Internet]. 2015 [citado 6 February 2017];22(2):35-38. Disponible en: http: //10.1016/s1405-8871(16)30044-x

42. Organización Mundial de la Salud. Recomendaciones Mundiales sobre Actividad Física Para la Salud [Internet]. 1st ed. Suiza: OMS; 2010 [citado 6 February 2017]. Disponible en: http://apps.who.int/iris/bitstream/ 10665/44441/1/9789243599977_spa.pdf

43. Yáñez A, Hespanhol J, Gómez R, Cossio M. Valoración de la actividad física en adolescentes escolares por medio de cuestionario. Rev Chil Nutrici [Internet]. 2014 [citado 20 Jun 2017]; 41(4):360-366. Disponible en: http://dx.doi.org/10.4067/ S0717-75182014000400003

44. González J, Portolés A. Actividad física extraescolar: relaciones con la motivación educativa, rendimiento académico y conductas asociadas a la salud. Rev Iberoameric Psicol Ejercic Depor [Internet]. 2013 [citado 20 Jun 2017]; 9(1):5165. Disponible en: http://www.redalyc.org/ html/3111/311130199005/ 
45. Romaní F, Gutierrez C. Auto-reporte de victimización escolar y factores asociados en escolares peruanos de educación secundaria, año 2007. Rev Peruan Epidemiol [Internet]. 2007 [citado 20 Jun 2017]; 14(3):201-209. Disponible en: http://www. redalyc. org/articulo.oa?id=203119676006

46. OMS. Fomento del consumo mundial de frutas y verduras [Internet]. Who. int. 2017 [actualizado 6 Feb 2017; citado 20 June 2017]. Disponible en: http:// www.who.int/dietphysicalactivity/fruit/es/ index1.html

47. Marquez U, Edinson R. Ingesta de frutas, verduras y sus motivaciones, barre- ras para consumir 5 porciones al día en los estudiantes de Nutrición de la Universidad Nacional Mayor de San Marcos [Licenciatura]. Universidad Nacional Mayor de San Marcos; 2015. Disponible en: http://cybertesis.unmsm.edu.pe/handle/ cybertesis/4296

48. Ferrante D, Linetzky B, Ponce M, Goldberg L, Konfino J, Laspiur S. Prevalencia de sobrepeso, obesidad, actividad física y tabaquismo en adolescentes argentinos: Encuestas mundiales de salud escolar y de tabaco en jóvenes, 2007-2012. Archiv Argent Pediat [Internet]. 2014 [citado 20 Jun 2017]; 112(6):500-504. Disponible en: http://dx.doi.org/10.5546/aap.2014.496 